\title{
The Stellar Population of the Galactic Bulge
}

\author{
M. Zoccali ${ }^{1}$ \\ ${ }^{1}$ Pontificia Universidad Católica de Chile \\ Casilla 306, Santiago 22, Chile \\ email: mzoccali@astro.puc.cl
}

\begin{abstract}
The Galactic bulge is the central spheroid of our Galaxy, containing about one quarter of the total stellar mass of the Milky Way $\left(\mathrm{M}_{\mathrm{bulge}}=1.8 \times 10^{10} M_{\odot}\right.$; Sofue, Honma \& Omodaka 2009). Being older than the disk, it is the first massive component of the Galaxy to have collapsed into stars. Understanding its structure, and the properties of its stellar population, is therefore of great relevance for galaxy formation models. I will review our current knowledge of the bulge properties, with special emphasis on chemical abundances, recently measured for several hundred stars.
\end{abstract}

Keywords. Galaxy: bulge, abundances, stellar content, formation

\section{The bulge structure}

The near infrared images from the COBE/DIRBE experiment clearly showed that our Galaxy has a boxy shaped bulge (Dwek et al. 1995). Isophote deprojection revealed a barlike nature, with axes ratios 1:0.33:0.23 and with the near side on the $1^{\text {st }}$ quadrant, at $\sim 20^{\circ}$ from the Sun-Galactic center direction. These findings were later confirmed by several authors (e.g., Babusiaux \& Gilmore 2005; Rattenbury et al. 2007a, and references therein), hence the prolate nature of the bulge is now widely accepted. The scale length of the main bar is $\sim 1.5 \mathrm{kpc}$. A smaller bar (scale $\sim 600 \mathrm{pc}$ ) seems to be also present in the inner bulge (Alard 2001, Nishiyama et al. 2005), though further studies are needed to confirm and characterize this structure.

A striking feature recently discovered in the outer bulge suggests that the Galactic bulge is all but a simple prolate spheroid. Along the minor axis, at distances in excess of $\sim 700 \mathrm{pc}$, a double red clump is clearly visible in several independent sets of data, symmetric both at positive and negative latitudes (McWilliam \& Zoccali 2009). The double clump disappears outside the minor axis, leaving only the brighter of the two at positive longitudes, and the fainter of the two at negative longitudes. Photometric data mapping the whole bulge area are presently available only from the 2MASS survey, which is not faint enough to reach the red clump for $|b|<3^{\circ}$. The Vista Variable in the Via Láctea survey (Minniti et al. 2009) will solve this problem, mapping the whole bulge to much fainter magnitudes ( $K_{s} \sim 20$ in the coadded images), and allowing to deproject its $3 \mathrm{D}$ structure by means of its RR Lyrae variable stars.

\section{The bulge age}

According to galaxy formation models, a bar can be formed through secular evolution of the disk. Dynamical instabilities would cause the bar to buckle (bend), then thicken and eventually look like a pseudobulge (Combes \& Sanders 1981, Combes et al. 1990, Athanassoula \& Misiriotis 2002, Athanassoula 2005). Deriving from disk stars, a pseudobulge would have several properties, such as kinematics, stellar ages, chemical 


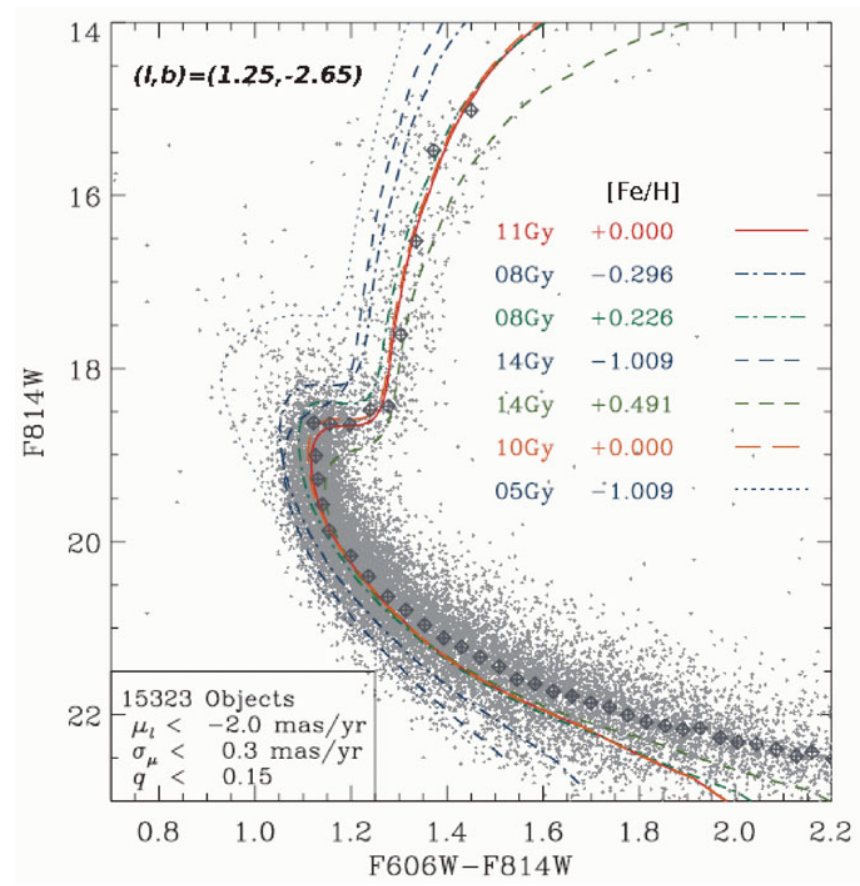

Figure 1. The CMD of a clean sample of bulge stars, selected on the basis of their proper motions. Also shown are isochrones for different ages and metallicities. It is evident that bulge stars follow the old isochrones, with the precise age depending on the adopted metallicity. The residual stars brighter than the old turnoff do not follow a younger isochrone, and indeed Clarkson et al. (2009) argues that they are likely to be all blue stragglers. Figure adapted from Clarkson et al. (2008).

abundances, resembling more those of galaxy disks rather than those of classical spheroids (Kormendy \& Kennicutt 2004).

In contrast with that, Ortolani et al. (1995) first demonstrated that the stellar population of the bulge, in Baade's Window at $(l, b)=(0,-4)$, is as old as the stars in the globular cluster 47 Tucanae. This result was later confirmed by Feltzing \& Gilmore (2000), Kuijken \& Rich (2002), Zoccali et al. (2003), and more recently by Clarkson et al. (2008) who excluded disk stars from the color magnitude diagram (CMD) on the basis of their proper motions (Fig 1). It should be noted, however, that all the above works studied the bulge stellar population in small fields very close to the minor axis. Dynamical simulation of bulge formation in the bar-driven scenario suggest that the vertical heating is significantly larger at the two ends of the bar, indicating these positions as the places where the intermediate age components should be found (e.g., Debattista et al. 2004; and references therein). Due to the larger interstellar extinction, deep photometry reaching the main sequence turnoff away from the minor axis is not yet available in the literature. Some data, acquired with the near IR camera HAWKI@VLT, are under analysis (Valenti et al. 2010). Brown et al. (2009) present a new photometric system employing five WFC3 bands spanning the UV, optical, and near IR, especially designed to break the degeneracy between reddening, temperature and metallicity, for the forthcoming WF3 Galactic bulge Treasury Program. This program will allow us to derive the bulge star formation history in four fields, including one at the far edge of the bar. 


\section{The bulge metallicity}

The mean age of the stars, as measured from the magnitude of the main sequence turnoff, is the most direct tool to date the formation of a stellar system. However, the sensitivity of the turnoff magnitude to age decreases for older stellar populations. The difference between two solar metallicity isochrones of 10 and $12 \mathrm{Gyr}$, respectively, is of $\Delta M_{V} \approx 0.18$ magnitudes, thus requiring a precision currently impossible to obtain for stars in the bulge, due to the instrinsic spread in distance, metallicity and differential reddening, all concurring to smear out the features of the CMD.

Luckily enough, the chemical composition of the stars has the opposite behaviour. Significant changes in the chemical composition of the interstellar medium, hence on the chemical composition of the newly born stars, occur during the first $\sim 1$ Gyr from the formation epoch of a stellar system. During that time, indeed, massive stars of different masses explode as core collapse supernovae ( $\mathrm{SNe}$ ), and later on the first thermonuclear SNe start exploding too, all enriching the medium of different kind of elements.

The bulge metallicity distribution function (MDF) was first determined by McWilliam and Rich (1994) who obtained high resolution $(\mathrm{R}=17,000)$ spectra of 11 bulge stars, and used them to calibrate a larger (88) sample of low resolution spectra from Rich (1988). They found a broad MDF, peaked at solar metallicity, with a shape roughly compatible with that of a closed box model. Mentioning here only works based on high resolution spectroscopy, a new determination of the MDF was made by Fulbright et al. (2006) using spectra for 27 stars observed at $\mathrm{R} \sim 60,000$ to re-calibrate a sample of 217 stars observed at low resolution by Sadler et al. (1996). The resulting MDF is very similar, only much smoothed, to the one by McWilliam \& Rich (1994). Both these studies were confined to the low extinction Baade's Window. Investigating the presence of a radial metallicity gradient in the bulge obviously requires the observations of many fields, which until a few years ago was only possible through photometry or low resolution spectroscopy. Minniti et al. (1995), using both their own and literature data, claimed the presence of a radial gradient, from $[\mathrm{Fe} / \mathrm{H}] \sim+0.2$ in Baade's Window $(\sim 600 \mathrm{pc})$, down to $[\mathrm{Fe} / \mathrm{H}] \sim-1$ at 2.3 kpc from the Galatic center, along the minor axis. Ramirez et al. (2000) and Rich et al. (2007a), however, claimed the absence of a gradient from Baade's Window inward. It is worth noticing that the work by Rich et al. (2007a) is based on high resolution near IR spectra, for a sample of $\sim 15$ stars in each of two fields at 140 and 600 pc, respectively.

The advent of the multifibre spectrograph FLAMES allowed a huge step forward in the field. Zoccali et al. (2008) acquired GIRAFFE spectra for $\sim 700$ bulge K giant stars in four different fields, containing 3 globular clusters. Spectra for about 200 red clump stars in Baade's Window, observed through the same setups and conditions, were added to the sample, from the french GIRAFFE GTO programme (Hill et al. 2009) The analysis of the stars in the fields along the bulge minor axis allowed to determine the MDF shown in Fig. 2, which is the first one derived from all high resolution spectra $(R=20,000)$. Clearly, the mean metallicity is higher in the innermost field, and decreases towards the outer one. It might also be noted that rather than a solid shift of the MDF towards the metal poor regime, going outwards the metal rich side becomes less and less populated, in favor of the metal poor one, thus suggesting something like a different proportion of populations at different radii. It should be emphasized that all three samples suffer from some degree of contamination from disk stars. The amount of disk contamination, at present, can only be estimated, e.g., using the Besançon Galaxy model (Robin et al. 2003 ), resulting in a fraction of $10 \%$ in the innermost field, $20 \%$ in the intermediate one and up to $50 \%$ in the outer one. This percentage, especially in the outer field, cannot be 


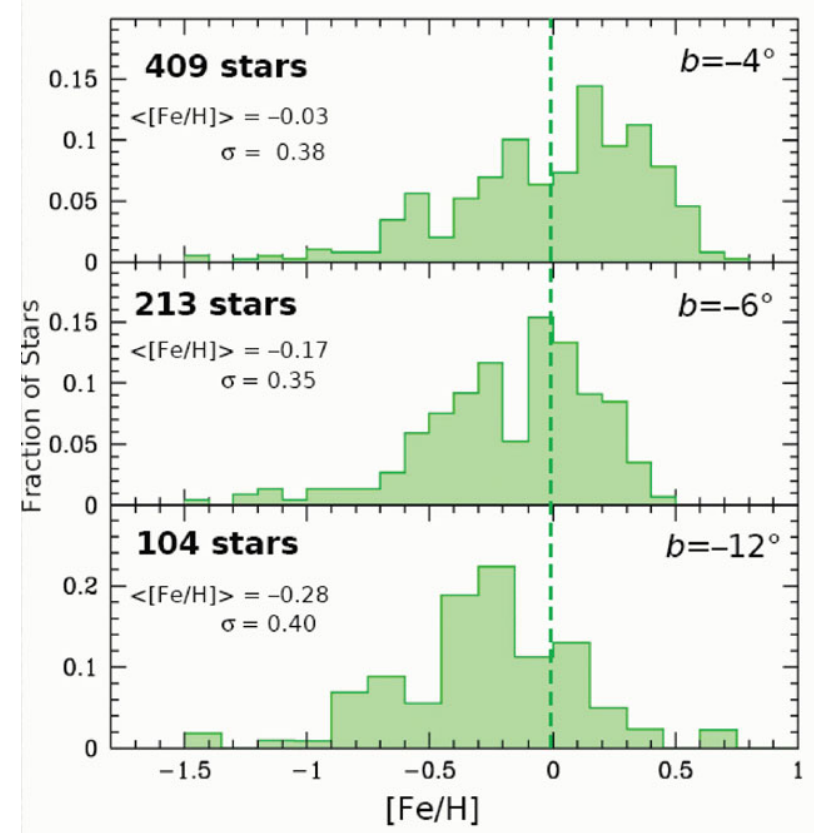

Figure 2. The bulge MDF in three fields along the minor axis, at latitudes listed on the top right corners. Mean metallicities and dispersions are labeled.

neglected and would obviously change the slope of the gradient. However it cannot erase the presence of a gradient (see Zoccali et al. 2008 for a discussion).

The bulge MDF in Baade's Window obtained by Zoccali et al. (2008) is centered at the same mean metallicity than the one by Fulbright et al. (2006). However, the former is significantly narrower than the latter, likely due to the smaller errors on the metallicity of individual stars. Recently Rangwala \& Williams derived the bulge MDF in a few fields including Baade's Window, by means of Fabry-Perot photometry across the Ca II triplet line at $8542 \AA$. Their MDF is compatible with both the Fullbright et al. (2006) and Zoccali et al. (2008) results, once the latter two are convolved with the larger error of the Fabry-Perot measurements.

The presence of a radial metallicity gradient along the bulge minor axis favors a bulge formation through dissipational collapse, against the bar-driven scenario. In fact, the latter is a dynamical process that should not, in principle, segregate metallicities. The independent evidence of the absence of a radial gradient in the inner region $(<600$ pc) cannot be contradicted here, thus one should keep in mind the possibility of a two component bulge, uniform in metallicity in its inner part, and with radially decreasing metallicity in the outer part.

Further evidence in the direction of a two component bulge comes from a detailed analysis of the MDF in Baade's Window. The observed MDF, once deconvolved from the estimated errors, appears clearly bimodal (Hill et al. 2009). Furthermore, the two components seems to have different kinematics (see below).

It is worth mentioning that in the past few years several high resolution spectroscopic analysis of bulge dwarf stars were carried on during microlensing events that temporarily magnifyied their brightness (Johnson et al. 2007, 2008; Cohen et al. 2008, 2009; Bensby et al. 2009a, 2009b). The results of these analysis were extremely surprising, because the mean $[\mathrm{Fe} / \mathrm{H}]$ of microlensed dwarfs was too high to be compatible with a random (if 
small) sampling of the bulge MDF obtained from giants. Newer results, however, now including a total of 13 bulge microlensed dwarfs, demonstrate that the initial discrepancy has almost completely disappeared, and it was very likely due to small number statistics (Bensby et al. 2009c, these proceedings).

\section{Bulge element ratios}

Element ratios carry important information about the formation timescale of a stellar system. In particular, the ratio of alpha elements over iron is a measure of the relative contribution of type II SNe (producing mainly alphas) relative to type Ia SNe (producing mainly iron). Therefore stars with $[\alpha / \mathrm{Fe}]$ significantly higher than 0 , such as the halo stars, were born before the lower mass type Ia SNe started to explode. McWilliam \& Rich (1994) first suggested that bulge stars have alpha element enhancement with respect to the Sun, suggesting a rapid star formation for the bulge. Rich \& Origlia (2005) confirmed this result with near IR spectra, though only for stars in a narrow range of $-0.35<[\mathrm{Fe} / \mathrm{H}]<0$. Zoccali et al. (2006) and Lecureur et al. (2007) extended the former studies to a sample of 50 stars, observed with UVES $(\mathrm{R}=45,000)$ simultaneously to the GIRAFFE observations mentioned above, and spanning $-0.8<[\mathrm{Fe} / \mathrm{H}]<+0.4$. Their results confirmed the alpha element enhancement of bulge stars, well reproduced by chemical evolution models assuming a rapid $(\sim 1 \mathrm{Gyr})$ star formation timescale (Immeli et al. 2004; Ballero et al. 2007).

Lecureur et al. (2007) found that different elements, such as oxygen and magnesium, behave differently, when plotted against $[\mathrm{Fe} / \mathrm{H}]$, thus supporting theoretical models with metallicity dependent stellar yields (c.f., McWilliam et al. 2008; Cescutti et al. 2009).

By comparing the $[\alpha / \mathrm{Fe}]$ trend of bulge $\mathrm{K}$ giant stars with that of solar neighborhood dwarfs (Bensby et al. 2004; Reddy et al. 2006) Zoccali et al. (2006) and Lecureur et al. (2007) concluded that the bulge is chemically different from the (local) thin and thick disks, and it must have formed more rapidly than both of them. These findings were confirmed by Cunha \& Smith (2006), Fulbright et al. (2007) and Rich et al. (2007a). More recently, Meléndez et al. (2008) questioned the above conclusions. By means of a homogeneous comparison of new near IR spectra of bulge $\mathrm{K}$ giants with similar data for thin and thick disk $\mathrm{K}$ giants, they found a similarity in the $[\mathrm{O} / \mathrm{Fe}]$ abundances of bulge and thick disk stars, supporting a similar origin -or at least formation timescale- for both components.

The origin of the different result of Meléndez et al. (2008) is the systematically higher $[\mathrm{O} / \mathrm{Fe}]$ they found for both thin and thick disk giants, while the bulge abundance ratios are consistent with all the previous results. They correctly emphasize the importance of a homogeneus comparison between the same kind of stars (K giants) in the bulge, thin and thick disk. Some of the previous studies (Fulbright et al. 2007; Rich et al. 2005, 2007a) also included spectra for disk $\mathrm{K}$ giants, analysed in the same way as the bulge ones, and yet yielded lower $[\mathrm{O} / \mathrm{Fe}]$ than bulge stars. However, the separation between thick and thin disk stars for this purposes must be done on the basis of kinematics only, it is very tricky, and it has not been discussed extensively in the papers mentioned above. Further investigation is needed in order to clarify whether the bulge and the thick disk do share the same element ratios, thus might have similar origin (e.g., Alves Brito et al. 2009, these proceedings).

The new, high resolution $(\mathrm{R}=70,000)$ near IR spectrograph CRIRES@VLT allowed Ryde et al. (2009) to obtain precise measurements of C,N,O elements in a sample of bulge $\mathrm{K}$ giants all included in the FLAMES-UVES sample observed by Lecureur et al. (2007). Their $[\mathrm{O} / \mathrm{Fe}]$ for bulge stars are compatible with most previous measurements, 
but have smaller statistical errors. The comparison with the thick disk giants observed by Meléndez et al. (2008) confirmed the chemical similarity between the two components.

It might be worth mentioning that high resolution near IR spectra have recently been obtained for a sample of red supergiant in the Galactic center $(<50 \mathrm{pc})$. All the available studies (Cunha et al. 2007; Davies et al. 2009; Najarro et al. 2009) agree on a [Fe/H] distribution sharply peaked around $\sim 0.1$; and on $[\mathrm{O} / \mathrm{Fe}]$ ratios evenly spread between +0.05 and +0.45 . The reason for alpha element enhancement in this case is rather unclear. The independent evidence of intense star formation in this region (e.g., An et al. 2009) implies recent formation of massive stars - thus core collapse SNe shortly after naturally enriching the inter stellar medium of oxygen and other alphas. Alternatively or simultaneously - it might also be that the recent star forming activity in the center was fueled by gas coming from the bulge/bar (known to produce gas inflows), hence already enriched by different kind of SNe and stellar winds, explaining the unusual element ratios.

\section{The bulge kinematics}

Several bulge proper motions studies have been carried on in different bulge windows, mostly for bright stars. Only in a few cases the photometry was deep enough to allow the kinematical decontamination of the turnoff region of the CMD ( Zoccali et al. 2001; Kuijken \& Rich 2002; Clarkson et al. 2008). Other studies aimed at the characterization of the bulge rotation and velocity dispersion, in order to understand if the bulge exibits a solid body rotation, if there are streaming motions, asymmetries, and if there is any evidence of different sub-populations with different kinematics (Spaenhauer et al. 1992; Alcock et al. 2001; Sumi et al. 2004; Rattenbury et al. 2007a,b; Vieira et al. 2007; Soto, Kuijken \& Rich 2007). Vieira et al. (2007, their Table 2) give a compilation of the available determinations of proper motions dispersions, all compatible within the errors with their $\sigma\left(\mu_{l}\right) \cos b=3.39 \pm 0.11 \mathrm{mas} / \mathrm{yr}$ and $\sigma\left(\mu_{b}\right)=2.91 \pm 0.09 \mathrm{mas} / \mathrm{yr}$.

Stellar kinematics can help understanding the nature of the Galactic bulge. In fact, it is expected that a pseudobulges formed through secular evolution of the disk will have a larger rotation compared to classical ones, and the rotation velocity is expected to be constant with galactic latitude. In the so-called Binney (1978) diagram, showing the ratio of the maximum rotation velocity over velocity dispersion, versus the asymmetry parameter, pseudobulges are expected to lie above classical ones. The galactic bulge, with its $V_{\max } / \sigma \sim 0.65$ (Rich et al. 2007b; Minniti \& Zoccali 2007) is consistent with classical spheroids in external galaxies. However, the latest results of the BRAVA survey (Howard et al. 2009) demonstrated that the rotation velocity of the bulge is constant with latitude (cilyndrical rotation), as expected for pseudobulges and opposed to a rotation velocity decreasing outwards, typical of classical spheroids (Combes et al. 1990; Fux 1997, 1999; Zhao et al. 1996; Athanassoula \& Misiriotis 2002; Athanassoula 2005)

Once more, the nature of the Galactic bulge seems consistent with either a classical or a pseudo bulge, depending on the tools used to probe it. A possible solution comes from the evidence that the metal rich and metal poor component have different kinematics, the first one more typical of a bar-like structure, the second of a dynamically hot system (Soto et al. 2007; Babusiaux et al. 2009).

\section{Conclusions}

The nature of the Galactic bulge is somehow puzzling. Its stellar population is old $(10-12$ Gyr $)$ and it has a metallicity distribution compatible with chemical enrichment 
models assuming a fast star formation. The abundance ratio of alpha elements over iron also supports a short star formation timescale, certainly more rapid than that of the thin disk, and possibly more rapid than that of the thick disk. The presence of a radial metallicity gradient, at least outside $\sim 600 \mathrm{pc}$, favors a formation scenario via dissipational collapse, rather than secular evolution of the disk. Nevertheless, the bulge has the shape of a bar (perhaps including some X-shape feature in the outer part) and a cylindrical rotation velocity, both characteristics of a pseudobulge formed via dynamical heating of a bar, resulting from disk secular evolution.

A possible solution to these conflicting results may come from the confirmation of a double component bulge, as already suggested by several studies (e.g., Soto et al. 2007, Hill et al. 2009, Babusiaux et al. 2009) and seen in several bulges of external galaxies (Peletier et al. 2007).

In any case, it is now clear from several independent evidences that the Galactic bulge is a complex structure. There is a metallicity gradient in the outer region that seems not to be present in the inner region. There are indications that the MDF in Baade's Window is bimodal, with each of the two component having different kinematics. Stellar ages are predicted to be different along the minor axis, compared to the edges of the bar. Even the morphology itself does not seem to be simply that of a bar, but rather something like an X-shape. The properties of the stellar population in Baade's Window cannot be considered as representative of the whole bulge: larger area photometric and spectroscopic maps are needed in order to understand the bulge structure and origin. The VVV survey, and its spectroscopic followups, will certainly reserve many surprises in this sense.

\section{Acknowledgements}

This work was supported by the Fondap Center for Astrophysics 15010003, CATA PFB-06, and Fondecyt Regular \#1085278.

\section{References}

Alard, C. 2001, $A \xi 3 A, 379$, L44

Alcock, C. et al., 2001, ApJ, 562, 337

An, D. et al. 2009, ApJ, 702, L128

Athanassoula, E. 2005, MNRAS, 358, 1477

Athanassoula, E. \& Misiriotis, A. 2002, MNRAS, 330, 35

Babusiaux, C. \& Gilmore, G. 2005 MNRAS, 358, 1309

Babusiaux, C. et al. 2009, $A \mathscr{E} A$, in preparation

Binney, J. 1978, MNRAS, 183, 501

Ballero, S. K., Matteucci, F., Origlia, L., \& Rich, R.M. 2007, A\&A, 467, 123

Bensby, T., Feltzing, S. \& Lundström, I. 2004, A\&A A, 421, 155

Bensby, T., Johnson, J. A., Cohen, J. G., et al. 2009a, A\&A, 499, 737

Bensby, T., Feltzing, S., Johnson, J. A. et al. 2009b, ApJL, 69, L174

Brown, T. M. et al. 2009, AJ, 137, 3172

Cescutti, G., Matteucci, F., McWilliam, A., \& Chiappini, C. 2009, A\&A, 505, 605

Clarkson, W. et al. 2008, ApJ, 684, 1110

Clarkson, W. et al. 2009, ApJ, submitted

Cohen, J. G., Huang, W., Udalski, A., Gould, A., \& Johnson, J. A. 2008, ApJ, 682, 1029

Cohen, J. G., Thompson, I. B., Sumi, T. et al. 2009, ApJ, 699, 66

Combes, F., Debbasch, F., Friedli, D., \& Pfenniger, D. 1990, A\&A, 233, 82

Combes, F. \& Sanders, R. H. 1981, A\& A, 96, 164

Cunha, K. \& Smith, V. V., 2006, ApJ, 651, 491 
Cunha, K. et al., 2007, ApJ, 669, 1011

Davies, B. et al. 2009, ApJ, 694, 46

Debattista, V. P., Carollo, C. M., Mayer, L., \& Moore, B. 2004 ApJL, 603, L25

Dwek, E. et al. 1995, ApJ, 445, 716

Feltzing S. \& Gilmore G. 2000 A\&A, 355, 949

Fulbright, J. P., McWilliam A., \& Rich R. M. 2006, ApJ, 636, 821

Fulbright, J. P., McWilliam, A., \& Rich, R. M. 2007, ApJ, 661, 1152

Fux, R. 1997, A\&SA, 327, 983

Fux, R. 1999, $A \& A, 345,787$

Hill, V. et al. 2009, $A \& A$, in preparation

Howard, C. D. et al. 2009, ApJL, 702, L153

Immeli A., Samland M., Gerhard O., \& Westera P. 2004, A\& A, 413, 547

Johnson, J. A., Gal-Yam, A., Leonard, D. C. et al. 2007, ApJ, 655, L33

Johnson, J. A., Gaudi, B. S., Sumi, T., Bond, I. A., \& Gould, A. 2008, ApJ, 685, 508

Kormendy, J. \& Kennicutt, R. C. Jr. 2004, ARA\&A, 42, 603

Kuijken, K. \& Rich, R. M. 2002, AJ, 124, 2054

Lecureur, A. et al. 2007, A\&A, 465, 799

McWilliam, A. \& Rich, R. M. 1994, ApJ, 91, 749

McWilliam, A. et al. 2008, AJ, 136, 367

McWilliam, A. \& Zoccali, M. 2009, ApJL, in preparation

Meléndez, J., et al. 2008, A\&̊A, 484, L21

Minniti, D. et al. 1995 MNRAS, 277, 1293

Minniti, D. \& Zoccali, M. 2007, in Bureau, M., Athanassoula, L. \& Barbuy, B. (eds.), Formation and Evolution of Galaxy Bulges, Proc. IAU Symposium No. 245 (Cambridge Univ. Press), p.1

Minniti, D. et al. 2009, Rev. Mexicana AyA, 35, 263

Najarro, F., Figer, D. F., Hillier, D. J., Geballe, T. R., \& Kudritzki, R. P. 2009, ApJ, 691, 1816

Nishiyama S. et al. 2005, ApJ, 621, 105

Ortolani S. et al. 1995, Nature, 377, 701

Peletier, R. et al. 2007, MNRAS, 379, 445

Ramírez, S. V., Stephens, A. W., Frogel, J. A., \& DePoy, D. L. 2000, AJ, 120, 833

Rangwala, N. \& Williams, T. B. 2009, ApJ, 702, 414

Rattenbury, N. J., Mao, S., Sumi, T., \& Smith, M. C. 2007a, MNRAS, 378, 1064

Rattenbury, N. J. et al. 2007b, MNRAS, 378, 1165

Reddy, B. E., Lambert, D. L., \& Allende Prieto, C. 2006, MNRAS, 367, 1329

Rich, R. M. 1988, AJ, 95, 828

Rich, R. M. \& Origlia, L. 2005, ApJ, 634, 1293

Rich, R. M., Origlia, L., \& Valenti E. 2007a, ApJ, 665, L119

Rich, R. M., Reitzel, D. B., Howard, C. D., \& Zhao, H. 2007b, ApJL, 658, L29

Robin, A.C., Reylé, C., Derrière S., \& Picaud S. 2003, A\&A, 409, 523

Ryde, N. et al. 2009, $A \& \mathcal{E} A$, in press (astro-ph/0910.0448)

Sadler, E. M., Rich, R. M., \& Terndrup, D. M. 1996, AJ, 112, 171

Sofue, Y., Honma, M., \& Omodaka, T. 2009, PASJ, 61, 227

Soto, M., Rich, R. M., \& Kuijken, K. 2007, ApJL, 665, L31

Spaenhauer, A., Jones, B. F., \& Whitford, A. E. 1992, AJ, 103, 297

Sumi, T. et al. 2004, MNRAS, 348, 1439

Valenti, E. et al. 2010 2010, $A \& A$, in preparation

Vieira, K. et al. 2007, AJ, 134, 1432

Zhao, H., Rich, R. M., \& Spergel, D. N. 1996, MNRAS, 282, 175

Zoccali, M. et al. 2001, AJ, 121, 2638

Zoccali M. et al. 2003, A\&A A, 399, 931

Zoccali, M. et al. 2006, $A \& A, 457, \mathrm{~L} 1$

Zoccali, M. et al. 2008, A\&A, 486, 177 